\title{
A HYPERSURFACE WHICH DETERMINES LINEARLY NON-DEGENERATE HOLOMORPHIC MAPPINGS
}

\author{
MANABU SHIROSAKI
}

\section{§1. Introduction}

In [S1] and [S2], the author gave hypersurfaces $S$ in $\boldsymbol{P}^{n}(\boldsymbol{C})$ with the property:

(UA) If two algebraically non-degenerate holomorphic mappings $f$ and $g$ of $\boldsymbol{C}$ into $\boldsymbol{P}^{n}(\boldsymbol{C})$ have the same pull-back $f^{*} S=g^{*} S$ as a divisor, then $f=g$.

However, the minimal degree of $S$ is of exponential order of $n$. In this paper, we give another hypersurface $S$ of much lower degree with the stronger property:

(UL) If two linearly non-degenerate holomorphic mappings $f$ and $g$ of $C$ into $\boldsymbol{P}^{n}(\boldsymbol{C})$ have the same pull-back $f^{*} S=g^{*} S$ as a divisor, then $f=g$.

\section{§2. Fundamental result}

We mean by a nonzero entire function an entire function with a point whose value is not zero. For two nonzero entire functions $f$ and $g$, we say that they are equivalent if the ratio $f / g$ is constant. This introduces an equivalence relation in each set of nonzero entire functions. The following theorem was given by Green $[\mathrm{G}]$ and Fujimoto $[\mathrm{F}]$ :

THEOREM A. Let $f_{0}, \ldots, f_{n}$ be nonzero entire functions such that $f_{0}^{d}+\cdots+$ $f_{n}^{d}=0$, where $d$ is a positive integer. If $d \geq n^{2}$, then

$$
\sum_{f_{j} \in I} f_{j}^{d}=0
$$

for each equivalence class I. Especially each class has at least two elements.

Now, we consider a homogeneous polynomial $P\left(w_{0}, w_{1}\right)$ of degree $d$ with the following property:

(U1) Let $f$ and $g$ be nonconstant holomorphic mappings of $\boldsymbol{C}$ into $\boldsymbol{P}^{1}(\boldsymbol{C})$ with representations $\tilde{f}=\left(f_{0}, f_{1}\right)$ and $\tilde{g}=\left(g_{0}, g_{1}\right)$, respectively. If $P\left(f_{0}, f_{1}\right)=$

Received April 8, 1999. 
$h^{d} P\left(g_{0}, g_{1}\right)$ holds for some meromorphic function $h$, then $f_{J}=\omega h g_{j}(0 \leq j \leq n)$, where $\omega^{d}=1$.

The existence of such polynomial is shown in [S2], where the minimal degree is 13 .

Definition. A holomorphic mapping $f$ of $\boldsymbol{C}$ into $\boldsymbol{P}^{n}(\boldsymbol{C})$ is linearly nondegenerate if its image is not contained in any hyperplane of $\boldsymbol{P}^{n}(\boldsymbol{C})$. This is equivalent to that $f_{0}, \ldots, f_{n}$ are linearly independent over $\boldsymbol{C}$, where $\left(f_{0}, \ldots, f_{n}\right)$ is a representation of $f$ in a homogeneous coorditate system of $\boldsymbol{P}^{n}(\boldsymbol{C})$.

\section{§3. Uniqueness of holomorphic mappings}

For a given $n$, we take an integer $q$ with $q \geq(2 n-1)^{2}$ and define a homogeneous polynomial $P_{n}\left(w_{0}, \ldots, w_{n}\right)$ of degree $d q$ by

$$
P_{n}\left(w_{0}, \ldots, w_{n}\right)=P\left(w_{0}, w_{1}\right)^{q}+P\left(w_{1}, w_{2}\right)^{q}+\cdots+P\left(w_{n-1}, w_{n}\right)^{q},
$$

and so, its minimal degree is $d(2 n-1)^{2}$ which is much smaller than $d^{n}$ of the minimal degree of the polynomials in [S1] and [S2].

Then, the hypersurface defined by the zero set of $P_{n}$ has the property (UL):

THEOREM. Let $f$ and $g$ be linearly non-degenerate holomorphic mappings of $\boldsymbol{C}$ into $\boldsymbol{P}^{n}(\boldsymbol{C})$ with representations $\tilde{f}=\left(f_{0}, \ldots, f_{n}\right)$ and $\tilde{g}=\left(g_{0}, \ldots, g_{n}\right)$, respectively. If

$$
P_{n}\left(f_{0}, \ldots, f_{n}\right)=\alpha P_{n}\left(g_{0}, \ldots, g_{n}\right)
$$

holds for an entire function $\alpha$ without zeros, then

where $\gamma^{d q}=\alpha$.

$$
f_{j}=\gamma g_{j} \quad(0 \leq j \leq n),
$$

Proof. By linear non-degeneracy, $P\left(f_{j}, f_{j+1}\right) \not \equiv 0$ and $P\left(g_{j}, g_{j+1}\right) \not \equiv 0$ and there are no equivalent pairs both in $\left\{P\left(f_{J}, f_{j+1}\right): 0 \leq j \leq n-1\right\}$ and in $\left\{P\left(g_{j}, g_{j+1}\right): 0 \leq j \leq n-1\right\}$. Hence, by Theorem A, there exist $k_{0}$ with $0 \leq k_{0}<$ $n$ and $\omega_{0}$ with $\omega_{0}^{q}=1$ such that

$$
P\left(f_{0}, f_{1}\right)=\omega_{0} \beta P\left(g_{k_{0}}, g_{k_{0}+1}\right),
$$

where $\beta$ is an entire function with $\beta^{q}=\alpha$. Also by Theorem $\mathrm{A}$, we have

$$
P\left(f_{1}, f_{2}\right)=\omega_{1} \beta P\left(g_{k_{1}}, g_{k_{1}+1}\right)
$$

for a $k_{1}$ with $0 \leq k_{1}<n, k_{1} \neq k_{0}$ and an $\omega_{1}$ with $\omega_{1}^{q}=1$. Fix an entire function $\gamma$ with $\gamma^{d}=\beta$. Then, by applying (U1) to (1) and (2), there exist $\eta_{0}$ and $\eta_{1}$ with $\eta_{0}^{d}=\eta_{1}^{d}=\omega_{0}$ such that

$$
f_{0}=\eta_{0} \gamma g_{k_{0}}, \quad f_{1}=\eta_{0} \gamma g_{k_{0}+1}
$$

and 


$$
f_{1}=\eta_{1} \gamma g_{k_{1}}, \quad f_{2}=\eta_{1} \gamma g_{k_{1}+1} \text {. }
$$

Hence, $\quad \eta_{0} g_{k_{0}+1}=f_{1} / \gamma=\eta_{1} g_{k_{1}}$. By linear non-degeneracy of $g$, we get $k_{0}<n-1, k_{1}=k_{0}+1$ and $\eta_{0}=\eta_{1}$. Therefore,

$$
P\left(f_{1}, f_{2}\right)=\omega_{0} \beta P\left(g_{k_{0}+1}, g_{k_{0}+2}\right) \text {. }
$$

is obtained. Successively, we have

$$
P\left(f_{J}, f_{J+1}\right)=\omega_{0} \beta P\left(g_{k_{0}+J}, g_{k_{0}+J+1}\right) \quad\left(j=0, \ldots, n-k_{0}-1\right) .
$$

By applying (U1) to this, as above, there exist $\eta_{j}$ with $\eta_{j}^{q}=\omega_{0}$ such that $f_{J}=\eta_{j} \gamma g_{k_{0}+\jmath}, f_{J+1}=\eta_{j} \gamma g_{k_{0}+\jmath+1}\left(j=0, \ldots, n-k_{0}-1\right)$. If $k_{0} \neq 0$, then there exist $m$ with $0 \leq m \leq k_{0}-1$ and $\omega^{\prime}$ with $\left(\omega^{\prime}\right)^{q}=1$ such that $P\left(f_{n-k_{0}}, f_{n-k_{0}+1}\right)=$ $\omega^{\prime} \beta P\left(g_{m}, g_{m+1}\right)$, and there exists $\eta^{\prime}$ with $\left(\eta^{\prime}\right)^{d}=\omega^{\prime}$ such that $f_{n-k_{0}}=\eta^{\prime} \gamma g_{m}$, $f_{n-k_{0}+1}=\eta^{\prime} \gamma g_{m+1}$. Hence, we get $\eta_{n-k_{0}-1} \gamma g_{n}=f_{n-k_{0}}=\eta^{\prime} \gamma g_{m}$, which is a contradiction because of $n \neq m$. Therefore we conclude $k_{0}=0$ and that

$$
f_{j}=\eta_{j} \gamma g_{j}, \quad f_{j+1}=\eta_{j} \gamma g_{j+1} \quad(j=0, \ldots, n-1) .
$$

These imply $\eta_{0}=\cdots=\eta_{n-1}$.

Q.E.D.

Remark. The hypersurface given in [S2] has Kobayashi hyperbolicity. However, our hypersurface is no longer Kobayashi hyperbolic for any $q$ if $n \geq 4$. In fact, a nonconstant holomorphic mapping $f=(\alpha: \zeta \alpha: 0: \beta: \xi \beta: 0: \cdots: 0)$ satisfies $f(\boldsymbol{C}) \subset S$, where $\alpha$ and $\beta$ are entire functions linearly independent over $C$, and $\zeta$ and $\xi$ are constants satisfying $P(1, \zeta)^{q}+P(\zeta, 0)^{q}=0$ and $P(0,1)^{q}+$ $P(1, \xi)^{q}=0$, respectively. Also, it is not difficult to prove Kobayashi hyperbolicity of $S$ for $q \geq(n-1)^{2}$ in the case of $n=2,3$.

\section{REFERENCES}

[F] H. Fujimoto, On meromorphic maps into the complex projective space, J. Math. Soc. Japan, 26 (1974), 272-288.

[L] M. L. GreEN, Some Picard theorems for holomorphic maps to algebraic varieties, Amer. J. Math., 97 (1975), 43-75.

[S1] M. SHIROSAKI, On polynomials which determine holomorphic mappings, J. Math. Soc. Japan, 49 (1997), 289-298.

[S2] M. SHIRosaki, On some hypersurfaces and holomorphic mappings, Koda1 Math. J., 21 (1998), 29-34.

Department of Mathmatical Sciences

COLLEGE OF ENGINEERING

Osaka Prefecture University

SAKAI 599-8531, JAPAN 